\title{
INTERMITTENT TINNITUS IN ADULTS: AN INSIGHT INTO COVERT HEARING LOSS
}

KEY WORDS: Intermittent \& continuous tinnitus, EHF thresholds, DPOAE.

\section{Badariya M.*}

Madhavan B. Afreen Najeeb C.H.

\section{Pooja Surendran}

Associate Professor, Department of Audiology and Speech Language Pathology, AWHSpecial College, Kallai, Calicut-673003. *Corresponding Author

Associate Professor, Department of Audiology and Speech Language Pathology, AWH Special College, Kallai, Calicut-673003.

Student, II MASLP, Department of Audiology and Speech Language Pathology, AWH Special College, Kallai, Calicut-673003.

Student, I MASLP, Department of Audiology and Speech Language Pathology, AWH Special College, Kallai, Calicut-673003.

Objectives: Association of high frequency hearing loss/minor damage in peripheral auditory system in continuous chronic tinnitus with normal PTA is well established. The purpose of the study was to verify whether this finding is true for intermittent unilateral or bilateral tinnitus patients with normal PTA using EHF audiometry and conventional DPOAEs. Materials and method: This study was conducted on 45 normal hearing adults between the age ranges of 18-30 years. Among them 30 adults comprised of study group with intermittent tinnitus which varies in laterality. Tinnitus evaluation was done on these population followed by THI administration. DPOAE and EHF audiometry was completed on all subjects after conventional hearing assessment program.

Result and Discussion: Kruskal Wallis H test \& Wilcoxon signed rank test was used to compare OAE amplitude \& EHF thresholds. Spearman's correlation was used to evaluate the correlation between DPOAE amplitude with EHF threshold. Reduced hearing sensitivity in the extended high frequency region may be early predictor of outer hair cell dysfunction in the most basal area. Findings of this study suggest that intermittent tinnitus may also lead to subtle lesion at the basal region of cochlea which would result in a significant hearing loss with continuous tinnitus in future.

Conclusion: Intermittent tinnitus may increase the fragility of peripheral auditory system which may lead to permanent lesions and would be evident as elevated thresholds in conventional PTA.

\section{INTRODUCTION}

Clinical evidence suggests that tinnitus is not homogenous; rather umbrella term tinnitus includes many subgroups. Tinnitus can be categorised as continuous, intermittent or temporary (Elouse A Koop, Fathima.T. Hussain 2019). In intermittent tinnitus, periods of presence and absence of the tinnitus alternate, and occur in a more or less periodic fashion (Humphriss, Hall, and Baguley 2016; Oiticica and Bittar 2015). Prevalence of intermittent tinnitus has not been well divulged into by previous researchers (Elouse A Koop, Fathima.T. Hussain 2019).

Association of subtle cochlear damage in continuous chronic tinnitus with normal Pure Tone Audiometry (PTA) is well established. From the audiological perspective, two tests are considered to be pertinent in detecting slight hearing alterations: otoacoustic emissions (OAE) and Extended High Frequency (EHF) audiometry of frequencies above $8,000 \mathrm{~Hz}$ (Sanchez TG et al., 2014).Various studies among adults using conventional pure tone audiometry has shown that the presence of tinnitus is often linked to changes in OAEs (Nieschalk et al., 1998; Sanchez et al., 2005; Sanches et al., 2010; Thabet et al., 2009). This reinforces that tinnitus may appear before hearing loss and that OAEs can detect minimal cochlear alterations in individuals with normal audiometry. Hence it is important to incorporate these two tests with conventional hearing assessment program in individuals with chronic tinnitus. Nevertheless the question remains whether this is true for chronic intermittent tinnitus population. One possible answer to approach this question is investigation, whether intermittent tinnitus also reflects in the same manner in EHF audiometry and DPOAE testing. The purpose of the study was to confirm whether intermittent tinnitus also points to hidden hearing loss/ basal cochlear lesions with normal PTA. Hence this study was undertaken. Aim of the study was to assess the presence of sub clinical OHC damage/subtle cochlear lesions in normal hearing individuals with intermittent tinnitus using EHF audiometry and DPOAEs.

\section{OBJECTIVES:}

1. Comparison of DPOAE amplitude and EHF threshold between normal hearing individuals with unilateral and bilateral intermittent tinnitus and control group.

2. To find correlation between DPOAE amplitude with EHF threshold across the groups.

\section{MATERIALS AND METHODS}

Participants:

Present study was conducted on 45 normal hearing adults between the age ranges of 18-30 years without gender preference. Subjects were divided into three groups of 15 subjects each. Participants of group one \& two were considered as study group with intermittent tinnitus which varies in laterality. Control group comprised age matched normal hearing adults. All participants fulfilling the following selection criteria were included in the study; like, dearth of noise exposure or ototoxicity, trauma; medical and other psychological complications in both the ears without hyperacusis. Bilateral normal otoscopic findings with PTA $\leq 20 \mathrm{~dB}$ HL at octave frequencies between $250 \mathrm{~Hz}$ to $8 \mathrm{KHz}$ and $>90 \%$ SIS scores at $40 \mathrm{dBSL}$. Normal middle ear functioning in both the ears, as tested by immittance evaluation. Individuals with pulsatile tinnitus were excluded from the study.

\section{Clinical characteristics of tinnitus of the study group}

Tinnitus duration: six months to one year, location of perception: unilateral as well as bilateral, frequency of perception: intermittent/temporary (once/twice in a week) and duration of each episode: less than an hour.

\section{Procedure\& Instrumentation:}

Tinnitus Handicap inventory was administered on subjects with tinnitus who fulfilled aforementioned criteria. Tinnitus evaluation was carried out on these subjects using MAICO MA 42 clinical audiometer with supra aural headphones. Two alternative forced choice pitch matching procedure was done and pitch of the tinnitus was determined according to the frequency of the signal that was matched with the tinnitus and 
this frequency was considered as reference for the loudness match. For loudness matching, a frequency that was matched to the patient's tinnitus was presented at a level just below the threshold and intensity increased until the subject signalled a match. A calibrated MAICO MA 42 clinical audiometer with supra aural headphones was used to obtain thresholds for conventional pure tone audiometry $(250 \mathrm{~Hz}$ to $8 \mathrm{KHz}$ ) and speech audiometry. Extended high frequency (10 to $16 \mathrm{KHz})$ thresholds were determined via Sennheiser HAD 300 circum aural headphones. Radio ear B-71 bone vibrator was used to determine bone conduction thresholds. A calibrated GSI Tympstar pro and MAICO ERO-SCAN OAE instrument was used for Immittance evaluations and to record DPOAEs bilaterally.

The DPOAE were recorded from both ears of each group using the MAICO ERO-SCAN in a sound proof room. DPOAEs were measured in the form of DP grams, with intensity of the two eliciting tones fixed between $1.6 \mathrm{khz}$ to $8 \mathrm{kHz}$ at $65(\mathrm{Ll})$ and $55 \mathrm{dBSPL}$ (L2) and with a frequency ratio F1/F2 of 1.22:1. DPs were considered to be present, only when the amplitude of DPOAE is greater than $-5 \mathrm{dBSPL}$ with a signal to noise ratio (SNR) exceeding 6dBSPL.Tympanometry was carried out using $226 \mathrm{~Hz}$ pure tone and reflexometry obtained for frequencies $500 \mathrm{~Hz}, 1 \mathrm{kHz}, 2 \mathrm{kHz}$ and $4 \mathrm{kHz}$. Modified Hughson and Westlake procedure was carried out to perform PTA and phonetically balanced PB words was used to estimate SIS.

The results were analysed using the Statistical Package for the Social Sciences (SPSS version 26.0).

\section{RESULTS}

Results of THI revealed, $73.3 \%$ of grouplindividual rated tinnitus as having slight handicap with high pitched tinnitus and reduced loudness in the right ear and $46.7 \%$ of group 2 with similar pitch \& loudness in both ears. Median \& quartile of DPOAE amplitude depicted better amplitude at mid frequencies but differences were not statistically significant across frequencies between groups.

Median and quartiles of EHF showed poorer thresholds towards high frequencies for both ears in individuals with tinnitus irrespective of tinnitus laterality.

Table 4: Median \& quartiles of extended high frequency thresholds.

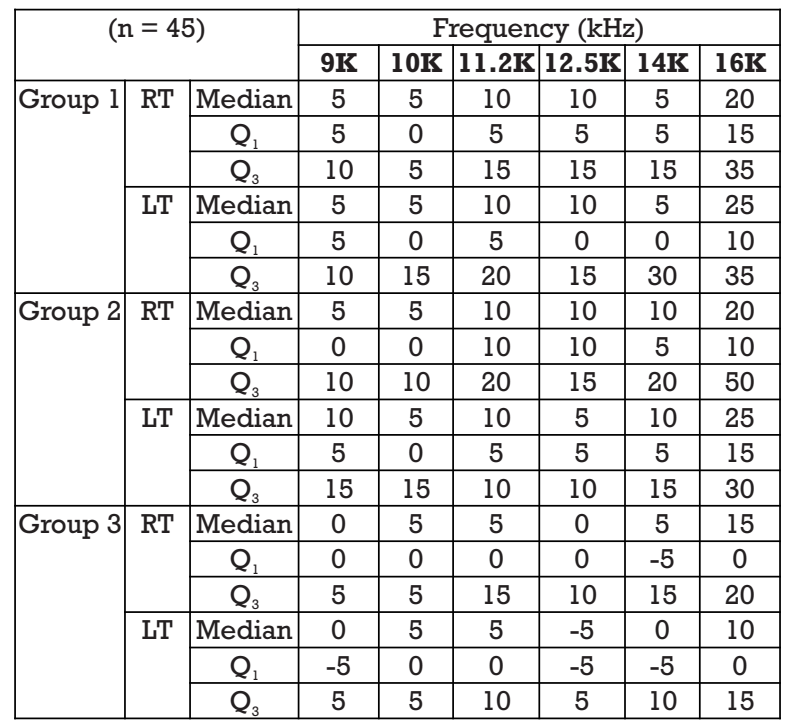

Results of Mann Whitney U test on extended high frequency threshold between group 1 \& control group revealed a statistically significant difference $(p=<0.05)$ in left ear. The same was true between bilateral tinnitus \& control group. But significant difference was absent between group one and two.
Table 5: Comparison of extended high frequency thresholds between individuals with unilateral tinnitus \& control group

\begin{tabular}{|c|c|c|c|c|}
\hline \multicolumn{5}{|c|}{ Group 1 \& 3 } \\
\hline & \multicolumn{2}{|c|}{ RT } & \multicolumn{2}{c|}{ LT } \\
\hline & $\mathbf{Z}$ & p value & Z & p value \\
\hline $9 \mathrm{KHz}$ & -1.787 & 0.074 & -2.53 & $0.011^{*}$ \\
\hline $10 \mathrm{KHz}$ & -0.313 & 0.754 & -2.176 & $0.030^{*}$ \\
\hline $11.2 \mathrm{KHz}$ & -1.517 & 0.129 & -2.321 & $0.020^{*}$ \\
\hline $12.5 \mathrm{KHz}$ & -2.62 & $0.009^{*}$ & -3.147 & $0.002^{*}$ \\
\hline $14 \mathrm{KHz}$ & -1.115 & 0.265 & -2.467 & $0.014^{*}$ \\
\hline $16 \mathrm{KHz}$ & -1.845 & 0.065 & -2.883 & $0.004^{*}$ \\
\hline
\end{tabular}

(*Significant)

The Mann Whitney U test was used to check whether the differences in extended high frequency threshold between individuals with unilateral tinnitus and control group were statistically significant or not, difference was present only at $12.5 \mathrm{KHz}$ in the right ear and all the frequencies at left ear. The same was repeated between bilateral tinnitus and control group and significant differences was present only in the left ear except at $10 \mathrm{KHz}$.

Table 6: Comparison of extended high frequency threshold between individuals with bilateral tinnitus \& control group

\begin{tabular}{|c|c|c|c|c|}
\hline \multicolumn{5}{|c|}{ Group 2 \& 3 } \\
\hline & \multicolumn{2}{|c|}{ RT } & \multicolumn{2}{c|}{ LT } \\
\hline & $\mathbf{Z}$ & p value & $\mathbf{Z}$ & p value \\
\hline $9 \mathrm{KHz}$ & -1.383 & 0.167 & -3.133 & $0.002^{*}$ \\
\hline $10 \mathrm{KHz}$ & -1.225 & 0.221 & -1.931 & 0.053 \\
\hline $11.2 \mathrm{KHz}$ & -2.182 & $0.029 *$ & -2.12 & $0.034^{*}$ \\
\hline $12.5 \mathrm{KHz}$ & -3.34 & $0.001^{*}$ & -3.287 & $0.001^{*}$ \\
\hline $14 \mathrm{KHz}$ & -1.727 & 0.084 & -2.507 & $0.012^{*}$ \\
\hline $16 \mathrm{KHz}$ & -1.713 & 0.087 & -3.298 & $0.001^{*}$ \\
\hline
\end{tabular}

To evaluate the correlation between DPOAE amplitude with EHF threshold across groups for both ears, Spearman's ratio was used. For this, median values of DPOAE amplitude across frequencies from $1.6 \mathrm{KHz}$ to $8 \mathrm{KHz}$ among and between groups were correlated with the median values of EHF threshold from $9 \mathrm{KHz}$ to $16 \mathrm{KHz}$ for both ears. Significant negative correlation was found across the groups for all EHFs except at $10 \mathrm{KHz}$ on both ears.

\section{DISCUSSION}

Most of the participants rated their tinnitus as having slight handicap on THI with high pitched tinnitus having a median value of $8 \mathrm{KHz}$ which was in accordance with literature Vernon and Meikle (2003), Gouveris et al., (2005) and Zhao et al., (2014).

In the present study, tinnitus laterality and elevated thresholds were evident (Vielsmeier V et al., 2015). This finding suggests that even intermittent tinnitus may be an indicator of subtle cochlear lesion at the basal region which would result in a significant hearing loss with continuous tinnitus in future. Absence of hyperacusis in the study group excluded the possibility of neural/maladaptive central gain origin of tinnitus (Hébert S. Fournier P,Noreña, A 2013).

To investigate the first objective of the study, absence of statistically significant difference in DPOAE amplitude could be due to reduced number of damaged outer hair cells in the study group which was not detectable through conventional DPOAE procedures. (Harding GW et al., 2004). Conventional DPOAE levels are useful for detecting broad OHC losses of greater than $10 \%$ and large focal OHC lesions greater than 0.6 $\mathrm{mm}$. Absence of significant differences in DPOAE amplitude across groups does not rule out the absence of subtle cochlear lesion in the study group. Similarly in the present study, OHC functioning beyond $8 \mathrm{KHZ}$ region in the cochlea could not be assessed as the DPOAEs were measured at frequencies from $1.6 \mathrm{kHz}-8 \mathrm{kHz}$. The study group might 
display differences in DPOAE amplitude if high frequency DPOAEs were employed.

Statistically significant EHF thresholds across groups verified the hypothesis that intermittent tinnitus might be an indicator towards subtle cochlear damage in the basal region. The study was able to identify major differences between the groups in extended high-frequency audiograms, suggesting subtle cochlear impairment in the tinnitus group. Elevated thresholds in the extended high frequency region may be early predictor of outer hair cell dysfunction in the most basal area. Tinnitus perception in the study group might be triggered by damage to the basal region of the cochlea. Lack of significant difference in EHF thresholds between individuals with unilateral and bilateral tinnitus could be due to the symmetry of tinnitus severity/tinnitus generation mechanism among the subjects in the study groups.

Significant negative correlation between DPAOE amplitude and EHF thresholds might be linked with decreased number of functioning OHCs beyond $8 \mathrm{KHz}$ region. As extended high frequency threshold worsens, the corresponding cochlear impairment also progresses systematically in a basal to apical direction (Osterhammel D, 1979). Thus present study suggests that EHF audiometry is a good tool to assess subclinical cochlear damage which affects the basal part of the cochlea initially. Result of this study indicate low efficiency of conventional DPOAE in detecting minor alterations in OHC functioning in the study population. DPOAEs at conventional frequencies lag in detecting damage confirmed with highfrequency audiometry (>8 kHz) (Reavis KM et al., 2008).

\section{SUMMMARY AND CONCLUSION}

To summarize, the results of the present study suggest that even temporary tinnitus is an early predictor of subtle progressive cochlear abnormalities. As well as increasing the vulnerability of the cochlea to further traumatic stimulation which was evident in extended high frequency audiometry. Thus the present study recommends, use of high frequency DPOAEs and EHF audiometry in individuals who complaint of chronic intermittent tinnitus along with conventional hearing assessment program.

In conclusion, extended high frequency audiometry can be considered as an early detector of cochlear abnormalities at the basal region of cochlea before the manifestation of hearing loss in conventional audiometry. Extended high frequency audiometry could be combined with high frequency DPOAE that specifically evaluate pre-neural peripheral function along with conventional DPOAE measures (Poling G et al., 2012) would disclose more valuable evidence to our hypothesis.

\section{REFERENCES}

1. Gouveris H, Maurer J, Mann W. DPOAE-grams in patients with acute tonal tinnitus. Otolaryngol Head Neck Surg. 2005; 132:550-553.

2. Harding GW, Bohne, BA, Ahmad M. DPOAE level shifts and ABR threshold shifts compared to detailed analysis of histopathological damage from noise. Hear. Res. 2002; 174:158-171.

3. Harding GW, Bohne BA. Temporary DPOAE level shifts, ABR threshold shifts and histopathological damage following below-critical-level noise exposures. Hear. Res. 2004; 196:94-108.

4. Hébert S. Fournier P, Noreña, $A$. The auditory sensitivity is increased in tinnitus ears.J.Neurosci. 2013;33:2356-2364.

5. Humphriss R, Hall AJ, Baguley DM. Prevalence and characteristics of spontaneous tinnitus in 11-year-old children.Int.J.Audiol.2016;55:142-148.

6. Koops EA, Husain FT, van Dijk P. Profiling intermittent tinnitus: a retrospective review. Int.J. Audiol. 2019;58:434-440.

7. Nieschalk M, Hustert B, Stoll W. Distortion-product otoacoustic emissions in middle-aged subjects with normal versus potentially presbyacusic highfrequency hearing loss. Audiology. 1998;37:83-99.

8. Oiticica J, Bittar RSM. Tinnitus prevalence in the city of São Paulo. Braz. J. Otorhinolaryngol.2015;81:167-176.

9. Osterhammel D, Osterhammel P. High-frequency audiometry: Age and sex variations. Scand.Audiol, 1979;8:73-80.

10. Poling G, Lee J, Siegel J, Dhar S. Clinical utilization of high-frequency DPOAEs. ENT Audiol. News. 2012;21:91-92.

11. Reavis KM, Phillips DS, Fausti SA, Gordon JS, Helt WJ, Wilmington D. et al., Factors affecting sensitivity of distortion-product otoacoustic emissions to ototoxic hearing loss. Ear Hear.2008;29:875-893.
12. Sanchez TG, Mak MP, Pedalini MEB, Levy CPD, Bento R F. Tinnitus and hearing evolution in normal hearing patients. Int. Arch. Otorhinolaryngol. 2005;9:220-7.

13. Sanches SGG, Sanchez TG, Carvallo RMM. Influence of cochlear function on auditory temporal resolution in tinnitus patients. Audiol. Neurotol. 2010; 15:273-281.

14. Sanchez TG, Oliveira JC, Kii MA, Freire K, Cota J, Moraes FVD. Tinnitus in adolescents: the start of the vulnerability of the auditory pathways. Codas. 2015;27:5-12.

15. Thabet EM. Evaluation of tinnitus patients with normal hearing sensitivity using TEOAEs and TEN test. Auris Nasus Larynx, 2009;36:633-636.

16. Vernon JA, Meikle MB.Tinnitus: clinical measurement. Otolaryngol. Clin. North Am. 2003;36:293-305,

17. Vielsmeier V, Lehner A, Strutz J, Steffens T, Kreuzer PM, Schecklmann M. et al., The relevance of the high frequency audiometry in tinnitus patients with normal hearing in conventional pure-tone audiometry. BioMed Res. Int. 2015.

18. Zhao F, Stephens SDG, Ishak WS, Meyer-Bisch C. The characteristics of Audioscan and DPOAE measures in tinnitus patients with normal hearing thresholds.Int.J.Audiol.2014;53:309-317. 